\title{
Cervical Spine Spondylitis with an Epidural Abscess in a Patient with Brucellosis:
}

\section{A Case Report}

\author{
Mohammad Reza Hasanjani Roushan ${ }^{1}$, Soheil Ebrahimpour ${ }^{1}$, Zeinab Mohseni Afshar², \\ Arefeh Babazadeh ${ }^{1 *}$ \\ 1 Infectious Diseases and Tropical Medicine Research Center, Health Research Institute, Babol University of Medical \\ Sciences, Babol, I.R. Iran \\ 2 Department of Infectious Diseases, Kermanshah University of Medical Sciences, Kermanshah, Iran
}

\begin{abstract}
Introduction: Human brucellosis, the most prevalent zoonotic disease worldwide, is a systemic infection which can involve several organs. Among musculoskeletal complaints, spondylitis is the most frequent complication of brucellosis and primarily affects the lumbar and thoracic vertebrae. The involvement of the cervical spine is infrequent.

Case report: This case report concerns an unusual case of cervical spine spondylitis with an epidural abscess due to Brucella in a 43-year-old man. The diagnosis was based on the patient being domiciled in an endemic region, his symptoms and his occupation. Clinical outcomes improved following antimicrobial therapy of rifampin, doxycycline, and gentamycin, and were confirmed radiologically.

Conclusion: Early diagnosis and treatment are crucial for these patients. The timely commencement of medical treatment can help prevent surgery.
\end{abstract}

Keywords: cervical spine spondylitis, brucellosis

Received: 29 December 2018 / Accepted: 15 May 2019

\section{INTRODUCTION}

Human brucellosis remains the world's most crucial bacterial zoonosis, with $>500,000$ new cases annually[1]. Infected animals excrete Brucella bacteria through the milk, urine, and the placenta. Human infection often results from direct contact with tissues from infected animals or by consumption of undercooked meat and unpasteurized dairy products. Human to human transmission has also been confirmed [2]. Brucellosis, is a systemic disease which can affect various organs [3], and may manifest as non-specific symptoms such as sweats, fatigue, back pain, and headache. The musculoskeletal system is commonly affected, and the infection can present as arthritis, sacroiliitis, spondylitis, spondylodiscitis, osteomyelitis, tenosynovitis, and bursitis [4]. Spondylitis and spondylodiscitis are the most common complications of brucellosis and primarily affect the lumbar and thoracic vertebrae [5]. Involvement of the cervical spine is very uncommon [6]. However, when occurring in the spinal area, compared to the lumbar area, the prognosis is worse with increased neurological complications.

Brucellosis should always be considered in the differential diagnosis of patients living in regions where the infection is endemic, and who present with musculoskeletal complaints.

A case of brucellosis with a cervical involvement is reported below.

\section{CASE REPORT}

A 43-year-old male farmer attended Rouhani Hospital, Babol University of Medical Sciences, after being referred by a family physician. He had been complaining of fever, night sweats, neck pain, and bilateral hand paresthesia for approximately three weeks previously.

No significant predisposing underlying disease or risk factor was reported at the time of admission. His temperature was $36.9^{\circ} \mathrm{C}$, pulse rate was 88 beats per minute, and blood pressure was $120 / 70 \mathrm{mmHg}$. His 
physical examination was notable for the following neurologic deficits.

Neck movements were restricted in all directions with a decreased degree of cervical lordosis. Muscle power of the deltoid muscle of both arms was $3 / 5$, and the triceps muscle of both arms was $4 / 5$. Other muscle powers and sense examinations were normal.

Concerning the evaluation of deep tendon reflexes, reflexes for both triceps muscles were decreased, though all other reflexes were normal. Neck stiffness, Kernig sign, Brudzinski, and bilateral Babinski signs were negative.

Laboratory tests were taken on admission and results were as follows: haemoglobin $(\mathrm{Hb}) 9.6 \mathrm{~g} / \mathrm{dL}$; white blood cells (WBCs) count $5.8 \times 10^{9} / \mathrm{L}$, platelet count $428 \times 10 \% / \mathrm{L}$; erythrocyte sedimentation rate (ESR) 62 $\mathrm{mm} / \mathrm{hr}$; C-reactive protein (CRP) $6 \mathrm{mg} / \mathrm{L}$; aspartate aminotransferase (AST) $55 \mathrm{U} / \mathrm{L}$; alanine aminotransferase (ALT) $15 \mathrm{U} / \mathrm{L}$; Wright's titre $1 / 80$; 2-mercaptoethanol (2ME) $1 / 40$ and Coombs Wright $1 / 80$. Moreover, two blood cultures, taken after forty-eight hours, were negative.

Magnetic resonance imaging (MRI) of the cervical spine showed an ill-defined intense enhancing mass at the anterior aspect of C6 and C7 vertebral borders with vertebral bodies enhancement and signal changes (low on T1, high on T2) at this level. Degenerating disc-bulging with effacing on the thecal sac was seen at C3/C4 to C5/C6 levels. Also, a mild compression effect on the spinal cord was noted (Fig.1). Additionally, a mild compression effect on the spinal cord was noted. (Fig. 1).

A tuberculin skin test (TST) for the patient was performed and no induration was seen after forty-eight hours and a chest radiogram was normal. Based on these results, spinal tuberculosis was not regarded as a leading differential diagnosis.

Osteoarticular TB with normal TST in an immunocompetent patient is an uncommon finding and pulmonary TB is not uncommon finding osteoarticular TB.

Regarding the sub-acute course of the patient's complaints, negative results of blood culture for pyogenic germs, negative TST, positive standard agglutination test for brucellosis, the MRI finding, and increased ESR, the conclusion arrived at was that the major differential diagnosis was either pyogenic spondylodiscitis (PS) or brucellosis, and tuberculous spondylodiscitis along with a related epidural abscess. Because of the patient's symptoms, brucellosis serology results, his occupation, and residence in endemic regions, it was decided to commence treatment for brucellosis.

A three-drug regimen composed of rifampin (Darou Pakhsh, Tehran, Iran) $600 \mathrm{mg}$ daily orally, doxycycline (Darou Pakhsh, Tehran, Iran) $100 \mathrm{mg}$ bid orally and gentamycin (Darou Pakhsh, Tehran, Iran) 240 mg daily, administered intramuscularly was prescribed. After two weeks, the patient's symptoms resolved entirely, and his paresthesia was reduced. Moreover, the size of the abscess decreased within a few weeks, as shown by MRI.

Treatment was continued for three months and the patient recovered progressively. His laboratory tests returned to within normal rangess and radiological follow-up was recommended.

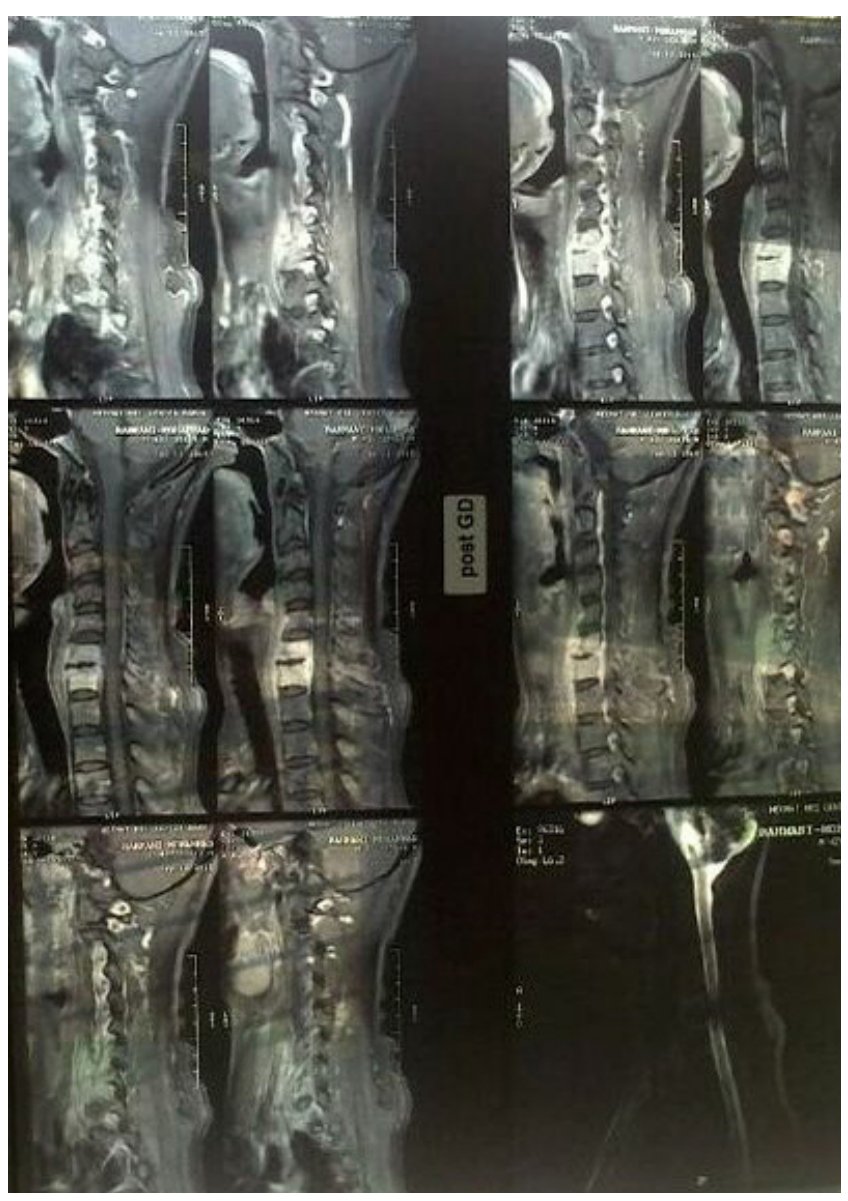

Fig. 1. Magnetic resonance imaging (MRI) of the cervical spine demonstrating an ill-defined intense enhancing mass at the anterior aspect of $\mathrm{C} 6$ and $\mathrm{C} 7$ vertebral borders with vertebral bodies enhancement and signal changes (low on T1, high on T2) at this level. Degenerating disc bulging with eroding of the thecal sac is seen at C3/C4 to C5/C6 levels. 


\section{DISCUSSION}

Brucellosis often presents with non-specific symptoms such as fever, chill, night sweating, lethargy, myalgia, and arthralgia. Musculoskeletal involvement is the most common manifestation of systemic brucellosis reported in 10-80\% of cases [7]. Muscle, joint and bone pain, and sometimes neurologic symptoms, such as power loss, paresthesia, and paraparesis can be seen in the cases with musculoskeletal involvements, as a result of these problems, our patient was referred to the health centre in an emergency [8]. The main physical examination findings of brucellosis have been reported as fever, hepatosplenomegaly (HSM) and at lower levels of lymphadenopathy. In the present case, the symptoms were fever, night sweating, restricted neck movements, decreased upper extremity strength and paresthesia. The lumbar spine is usually affected, but the involvement of the cervical spine is infrequent [9]. The clinical significance of cases of cervical spondylitis together with an epidural abscess is that there is increased neurological complications and a poor prognosis. It is challenging to diagnose brucellar spondylitis with an epidural abscess which cause nonspecific symptoms such as lower back and neck pain, fever, and malaise. Concerning the diagnosis of brucellar spondylitis, the serological tests, radiology exams, medical history, and clinical findings are important and worthwhile [10]. The most valuable radiological technique in the diagnosis of spinal brucellosis and the spinal epidural abscess is MRI due to its high- resolution power and ability to characterise tissues. It is used for early diagnosis and in the follow-up of treatment outcome. In this case, the marked signal increase in the bodies of the $\mathrm{C} 6$ and $\mathrm{C} 7$ vertebrae and enhancing abscess formation in the epidural area were detected on MRI. Contrast-enhanced MRI should be preferred to assess for any improvement [11]. Brucellosis is diagnosed with laboratory tube agglutination tests above $1 / 320$, titre 2 -mercaptoethanol $(2 \mathrm{ME}) \geq 1: 160$, and growth of the organism in blood culture [12]. However, in the present case, the result of the Wright test was lower than the diagnostic threshold, and blood culture was negative for Brucella. However, because of the patient's symptoms, his residency in an endemic area, and his occupation, it was decided to treat for brucellosis.

Infectious diseases, malignancy, and complications due to trauma should be considered in the differential diagnosis. Tuberculosis (TB) spondylitis should be a primary consideration if strong clinical suspicion ex- ists. Radiological changes in musculoskeletal TB resemble brucellosis. However, in contrast to brucellosis, which usually involves the lumbar spine, it causes thoracic involvement. In $\mathrm{TB}$ involvement, the changes in the affected vertebra start earlier and are more prominent, with the occurrence of severe damage, collapse, large paraspinal abscesses, and gibbus deformity [13].

Furthermore, vertebral metastases, degenerative changes, hematoma, and trauma should also be considered in the differential diagnosis. The studies emphasise that treatment of brucellosis should be continued until the ESR and CRP return to the normal levels and the treatment period should be at least 3-6 months in cases with an abscess [14]. Treatment options for spinal brucellosis include antibiotic therapy and surgery. Treatment duration of brucellar spondylitis is much longer than systemic brucellosis, and long-term treatment aims to prevent relapse.

The rate of surgical intervention ranges from $7.6 \%$ to $33 \% \%$ in brucellar spondylitis. Surgery is preferred in cases such as the progressive neurological deficit, spinal instability, vertebral collapse and following failure of antibiotic therapy.

In the current case, fortunately, the medical treatment led to a complete recovery with progressive reduction in the abscess size.

A standardized therapy for brucellar spondylitis has not been defined, but treatment duration of at least three to six months is suggested $[15,16]$. A triple combination of doxycycline and rifampin for three months with gentamycin added in the first weeks of treatment was prescribed in the present case. Surgery was not required because the patient responded well to this therapeutic regime.

\section{CONCLUSIONS}

An abscess is a serious complication of brucellar spondylitis that can lead to permanent damage by causing neurological deficits. Early diagnosis and treatment are crucial in patients with symptoms such as fever, lethargy, paravertebral muscle spasm, lower back, and neck pain. Treatment must be determined according to the severity of the disease and the patient's clinical condition. In cases where infections that do not respond to antibiotic therapy and especially when progressive neurological damages occur, surgery is the alternative treatment mode. 


\section{ACKNOWLEDGEMENTS}

The authors thank the Department of Internal Medicine of Babol University of Medical Sciences, Iran.

\section{- CONFLICT OF INTEREST}

None to declare.

\section{REFERENCES}

1. Herrick JA, Lederman RJ, Sullivan B, Powers JH, Palmore TN. Brucella arteritis: clinical manifestations, treatment, and prognosis. Lancet Infect Dis. 2014;14(6):520-6.

2. Tuon FF, Gondolfo RB, Cerchiari N. Human-to-human transmission of Brucella - a systematic review. Trop Med Int Health. 2017;22(5):539-46.

3. Hasanjani MR, Bayani M, Soleimani SA, Mohammadnia-Afrouzi $\mathrm{M}$, Nouri H, Ebrahimpour S. Evaluation of CD4+ CD25+ FoxP3+ regulatory $T$ cells during treatment of patients with brucellosis. J Biol Regul Homeost Agents. 2016;30(3):675-82.

4. Ebrahimpour S, Bayani M, Moulana Z, Hasanjani Roushan MR. Skeletal complications of brucellosis: A study of 464 cases in Babol, Iran. Caspian J Intern Med. 2017;8(1):44-8.

5. Kutlu SS, Kutlu M, Tuzun T, Ozdemir K. Spondylodiscitis: a common complication of brucellosis. J Infect Dev Countries. 2018;12(07):550-6.

6. Lampropoulos C, Kamposos P, Papaioannou I, Niarou V. Cervical epidural abscess caused by brucellosis. BMJ Case Rep. 2012;2012:bcr2012007070.
7. Reşorlu H, Saçar S, Inceer BŞ, et al. Cervical spondylitis and epidural abscess caused by brucellosis: a case report and literature review. Folia Med. 2016;58(4):289-92.

8. Turan H, Serefhanoglu K, Karadeli E, Togan T, Arslan H. Osteoarticular involvement among 202 brucellosis cases identified in Central Anatolia region of Turkey. Intern Med. 2011;50(5):421-8.

9. Lim K-B, Kwak Y-G, Kim D-Y, Kim Y-S, Kim J-A. Back pain secondary to Brucella spondylitis in the lumbar region. Ann Rehabil Med. 2012;36(2):282-6.

10. Yang B, Hu H, Chen J, He X, Li H. The Evaluation of the Clinical, Laboratory, and Radiological Findings of 16 Cases of Brucellar Spondylitis. BioMed Res Int. 2016;2016:6.

11. Ural O, Sümer \$̧, Demir NA, Dikici N, Firat V. Isolated Cervical Spondylodiscitis Due to Brucellosis: A Case Report. J Physic Med Rehabil Sci. 2013;16(3).

12. Hasanjani Roushan MR, Ebrahimpour S. Human brucellosis: An overview. Caspian J Intern Med. 2015;6(1):46.

13. Garg RK, Somvanshi DS. Spinal tuberculosis: a review. J Spinal Cord Med. 2011;34(5):440-54.

14. Gangi SMS, Hasanjani Roushan MR, Janmohammadi N, Mehraeen R, Amiri MJS, Khalilian E. Outcomes of treatment in 50 cases with spinal brucellosis in Babol, Northern Iran. J Infect Dev Countries. 2012;6(09):654-9.

15. Lee HJ, Hur JW, Lee JW, Lee SR. Brucellar spondylitis.J Korean Neurosurg Soc. 2008;44(4):277-9.

16. Hasanjani Roushan MR, Gangi SMS, Janmohammadi N. Update on the treatment of adult cases of human brucellosis. Arch Clin Infect Dis. 2008;3(3):167-73. 